\title{
BMJ Open Acupuncture for patients with chronic urticaria: a systematic review protocol
}

\author{
Qin Yao, ${ }^{1,2}$ Yongming Ye, ${ }^{1}$ Xiaoxu Liu, ${ }^{1,2}$ Zongshi Qin, ${ }^{1,2}$ Zhishun Liu ${ }^{1}$
}

To cite: Yao Q, Ye Y, Liu X, et al. Acupuncture for patients with chronic urticaria: a systematic review protocol. BMJ Open 2015;5: e007704. doi:10.1136/ bmjopen-2015-007704

- Prepublication history and additional material is available. To view please visit the journal (http://dx.doi.org/ 10.1136/bmjopen-2015007704)

Received 16 January 2015 Revised 25 May 2015 Accepted 7 July 2015

\section{CrossMark}

${ }^{1}$ Department of Acupuncture, Guang'anmen Hospital, China Academy of Chinese Medical Sciences, Beijing, China

${ }^{2}$ School of Graduates, Beijing University of Chinese Medicine, Beijing, China

Correspondence to Professor Zhishun Liu; liuzhishun@aliyun.com

\section{ABSTRACT}

Introduction: This systematic review protocol aims to describe a meta-analysis to assess the effectiveness and safety of acupuncture therapy for patients with chronic urticaria.

Methods and analysis: We will electronically search the following databases: the Cochrane Central Register of Controlled Trials (CENTRAL), PubMed, EMBASE, the Web of Science, Traditional Chinese Medicine, China National Knowledge Infrastructure (CNKI), Chinese Biomedical Literature Database (CBM), Chinese Scientific Journal Database (VIP database) and WanFang Database from their inception. Other sources will also be searched, including the reference lists of identified publications and conference proceedings. Any clinical randomised controlled trials related to acupuncture for treating chronic urticaria without restriction of publication status and languages will be included. Study selection, data extraction and assessment of study quality will be performed independently by 2 researchers. Data will be synthesised by either the fixed-effects or randomeffects model according to a heterogeneity test. Improvement in pruritus and wheals will be assessed as the primary outcomes. Adverse events, quality of life and the recurrence rate after at least 3 months of the treatment will also be evaluated as secondary outcomes. RevMan V.5.2 statistical software will be used for meta-analysis, if possible. If it is not appropriate for a meta-analysis, then a descriptive analysis or a subgroup analysis will be conducted. The results will be expressed as a risk ratio for dichotomous data and mean difference (MD) or standardised MD for continuous data.

Dissemination and ethics: The protocol of this systematic review will be disseminated in a peerreviewed journal and presented at relevant conferences. It is not necessary for a formal ethical approval because the data are not individualised.

Trial registration number: PROSPERO CRD42015015702.

\section{INTRODUCTION}

\section{Description of the condition}

Urticaria is a heterogeneous group of diseases involving the onset of pruritic wheals, angio-oedema or both. ${ }^{1-4}$ Chronic urticaria is diagnosed when recurrent crops of

\section{Strengths and limitations of this study}

- There is currently one systematic review on 'acupuncture and moxibustion' and 'chronic urticaria', published in 2009. Our review will assess the effectiveness and safety of acupuncture therapy for patients with chronic urticaria.

- Chronic urticaria is difficult to treat. The results of the systematic review could help clinicians make decisions about possible treatments for chronic urticaria.

- The different criteria for efficacy evaluation and forms of acupuncture therapies may cause significant heterogeneity in this review.

urticaria continue for more than 6 weeks. ${ }^{2}$ It is a common disease with $0.5-1 \%$ prevalence, and nearly $20 \%$ of people suffer from urticaria at least once during their lifetime. ${ }^{5}$ Women suffer from urticaria nearly twice as often as men. Individuals between 20 and 40 years have a greater chance of suffering from chronic urticaria. ${ }^{5}$

Chronic urticaria negatively influences the patient's quality of life because of the itching or physical discomfort during outbreaks. The severity of urticaria varies between individuals. One study found that outbreaks lasted 6-10 weeks in $58 \%$ of respondents, while $12 \%$ of patients had outbreaks lasting 52 weeks per year. Patients suffering from chronic urticaria have their sleep affected an average of three times per week. ${ }^{6}$ The disease also has a large impact on society because of its high direct and indirect healthcare costs. ${ }^{2} 7$

Chronic urticaria can be divided into chronic spontaneous urticaria and chronic inducible urticaria. Depending on the underlying causes, which are varied and complex, chronic inducible urticaria is divided into physical urticaria (including symptomatic dermographism, cold-induced urticaria, pressure urticaria, solar urticaria, heat-induced urticaria and vibration-induced angio-oedema), cholinergic urticaria, contact urticaria or aquagenic urticaria. However, one patient 
may have several subtypes of urticaria at once. The most important diagnostic step of chronic urticaria includes a thorough history, physical examination and a ruling out of severe systemic disease. A thorough history should include most possible inducible factors or critical aspects of the nature of the patient's urticaria. There is no specific serum test for chronic urticaria. The autologous serum skin test may be used for non-specific autoantibodies against either IgE or the high-affinity IgE receptor, $^{8}$ and there are some specific provocations that may be used to test underlying causes.

The management of urticaria is aimed at alleviating symptoms. The first line includes elimination of the underlying causes and eliciting triggers. The second line includes symptomatic treatment with pharmacotherapy. ${ }^{2}{ }^{10}$ The first-line treatment is non-sedating antihistamines. If the first-line treatment is not effective after a maximum of 2 weeks, increasing the dosage up to fourfold is recommended. Second-line therapies should be added to the antihistamine treatment when patients do not respond to a fourfold increase in dosage. Short-term corticosteroids may be prescribed for exacerbations. No serious adverse events or death events were reported in studies according to some relevant studies when participants were taking H1-antihistamines. ${ }^{11-14}$ The side effects of H1-antihistamines, which include headache, somnolence, fatigue, dry mouth, hay fever, allergies, etc, were reported in some studies. $^{12} 14$

\section{Description of the intervention}

Acupuncture, which has been used for over 2000 years, is an important part of traditional Chinese medicine (TCM) and complementary and complementary medicine. Acupuncture therapy has very few adverse effects and is widely used for the treatment of urticaria in Asia. ${ }^{15} 16$ Many clinical studies have reported that acupuncture is effective for the treatment of urticaria. ${ }^{15}$

\section{How the intervention might work}

In TCM, acupuncture is believed to function by regulating the balance of Qi circulation. In Western medicine, the mechanism of acupuncture is yet not clear. It is speculated that the potential mechanism of acupuncture for treating urticaria might be the suppression of the immunological reaction. ${ }^{17}{ }^{18}$ Studies in animals and humans signified that acupuncture can attenuate histamine effect, modulate the function of the immune system and decrease the adhesion molecule. ${ }^{19} 20$

\section{Why it is important to do this review}

So far, there is only one systematic review published in Chinese investigating acupuncture and chronic urticaria. $^{21}$ The previous review included 12 randomised controlled trials (RCTs), but only 4 of those studies strictly investigated the effectiveness of acupuncture. Additionally, the outcome assessments were not clearly or specifically described. Therefore, no definite conclusions on the effectiveness of acupuncture for chronic urticaria can be drawn. There have been at least four more RCTs reported in the past 5 years. Using a stricter search strategy, a more objective outcome evaluation and a rigorous review method, we expect that our systematic review will provide a more convincing conclusion.

\section{Objectives}

To systematically evaluate the effectiveness and safety of acupuncture therapy for patients with chronic urticaria.

\section{METHODS AND ANALYSIS \\ Inclusion criteria for study selection \\ Types of studies}

All RCTs without restrictions on publication status will be included. Quasi-randomised trials will be excluded.

\section{Types of participants}

Patients with chronic urticaria, regardless of sex, age, race or educational and economic status, according to the following criteria:

1. EAACI/GA2LEN/EDF/WAO guideline: definition, classification and diagnosis of urticaria. ${ }^{1-4} 9$

2. Chinese guidelines for the diagnosis and treatment of urticaria. ${ }^{22} 23$

\section{Types of interventions}

Acupuncture is defined as the stimulation of acupuncture points by needles, including manual acupuncture, dermal needle, plum blossom needle, ear acupuncture, electroacupuncture or fire needle. Other stimulation methods including acupressure, moxibustion, laser acupuncture, pharmacoacupuncture, dry needling or transcutaneous electrical nerve stimulation will be excluded. Sham acupuncture includes sham acupuncture at selected acupoints, sham acupuncture at non-acupoints, needling at inappropriate acupoints, non-penetrating sham acupuncture and pseudo interventions). ${ }^{24}$ The following treatment comparisons will be investigated.

Acupuncture compared with no treatment.

1. Acupuncture compared with placebo or sham acupuncture.

2. Acupuncture compared with other active therapies.

3. Acupuncture in addition to active therapy compared with the same active therapy.

We will exclude RCTs in which one form of acupuncture was only compared with another form of acupuncture or a different type of TCM (eg, Chinese herbal medicine). We will assess the acupuncture treatment according to how the acupuncturists are trained and educated, their clinical experience, total numbers of acupuncture sessions, treatment duration and treatment frequency, etc. ${ }^{25}$ 
Types of outcome measures

\section{Primary outcomes}

Improvement in pruritus and wheals, using urticaria activity score (UAS) ${ }^{26}$ or other validated scales, at least after 2 weeks of treatment.

\section{Secondary outcomes}

Secondary outcomes include adverse events and quality of life using a health-related quality of life measure, including a generic, dermatology-specific or diseasespecific instrument. ${ }^{10} 27$ The recurrence rate after at least 3 months of the treatment will also be evaluated.

\section{Search methods for identification of studies \\ Electronic searches}

We will electronically search the following databases, regardless of publication status and languages: the Cochrane Central Register of Controlled Trials (CENTRAL), PubMed, EMBASE, the Web of Science, Traditional Chinese Medicine databases, China National Knowledge Infrastructure (CNKI), Chinese Biomedical Literature Database (CBM), Chinese Scientific Journal Database (VIP database) and Wan-Fang Database from their inception. The following search terms will be used: chronic urticaria, hives, nettle-rash, Fong-Tzen-Kwai, wind-rash-patch, acupuncture, manual acupuncture, filiform steel needle, electroacupuncture, fire needling, auricular acupuncture, ear acupuncture, dermal needle, abdominal acupuncture, pyonex and plum blossom needle. The equivalent search words will be used in the Chinese databases. A search strategy created according to the Cochrane handbook guidelines will be conducted in all the electronic databases. ${ }^{28}$ The search strategy for PubMed is shown in table 1.

\section{Searching other resources}

We will scan the reference lists of identified publications for additional trials. We will search PubMed, Turning Research Into Practice (TRIP) database and the Cochrane Library for existing systematic reviews possibly relevant to this systematic review to search their reference lists for additional trials. We will also search conference proceedings related to this topic.

\section{Data collection and analysis}

Selection of studies

We plan to conduct this systematic review between 1 January 2015 and 30 August 2015. All reviewers have undergone training to ensure a basic understanding of the background and purpose of the review. After electronic searching, the records will be uploaded to a database set up by EndNote software (V.X7). Records selected from other sources will also be moved to the same database. Two review authors ( $Q Y$ and $\mathrm{ZQ}$ ) will independently screen the titles, abstracts and keywords of all retrieved studies and decide which trials meet the inclusion criteria. We will obtain the full text of all possibly relevant studies for further assessment. Excluded
Table 1 Search strategy used in PubMed

\begin{tabular}{|c|c|}
\hline No & Search items \\
\hline 1 & Randomised controlled trial.pt \\
\hline 2 & Controlled clinical trial.pt \\
\hline 3 & Randomised.ti,ab \\
\hline 4 & Randomly.ti,ab \\
\hline 5 & Placebo.ti,ab \\
\hline 6 & Trial.ti,ab \\
\hline 7 & Groups.ti,ab \\
\hline 8 & 1 or $2-7$ \\
\hline 9 & Urticaria.Mesh \\
\hline 10 & Chronic urticaria.ti,ab \\
\hline 11 & hives.ti,ab \\
\hline 12 & nettle-rash.ti,ab \\
\hline 13 & Angioedema.ti,ab \\
\hline 14 & Fong-Tzen-Kwai.ti.ab \\
\hline 15 & wind-rash-patch.ti.ab \\
\hline 16 & 9 or $10-15$ \\
\hline 17 & Acupuncture therapy. Mesh. \\
\hline 18 & Acupuncture.ti,ab \\
\hline 19 & Acupoints.ti,ab \\
\hline 20 & Acupuncture*.ti,ab \\
\hline 21 & Body acupuncture.ti,ab \\
\hline 22 & Scalp acupuncture.ti,ab \\
\hline 23 & manual acupuncture.ti,ab \\
\hline 24 & Auricular acupuncture.ti,ab \\
\hline 25 & ear acupuncture.ti,ab \\
\hline 26 & Electroacupuncture.ti,ab \\
\hline 27 & Fire needling.ti,ab \\
\hline 28 & dermal needle.ti,ab \\
\hline 29 & plum blossom needle.ti,ab \\
\hline 30 & Pyonex.ti,ab \\
\hline 31 & Abdominal acupuncture.ti,ab \\
\hline 32 & Filiform steel needle.ti,ab \\
\hline 33 & 17 or $18-32$ \\
\hline 34 & 8 and 16 and 33 \\
\hline
\end{tabular}

studies will be recorded with explanations. Any disagreements will be resolved by discussion between the two authors (QY and $\mathrm{ZQ}$ ) and the third author (ZL) for arbitration when necessary. We will contact authors of trials for clarification when necessary. The study flow diagram is shown in figure 1 .

\section{Data extraction and management}

Two authors will independently extract data from the selected reports or studies and fill in the data extraction form. We will obtain data for general information, participants, methods, interventions, outcomes, results, adverse events, conflicts of interest, ethical approval and other information. We will contact the authors for further information when the reported data are not sufficient. Any disagreements will be resolved by discussion between the two authors, and further disagreements will be arbitrated by the third author (ZL).

\section{Assessment of risk of bias in included studies}

Two authors (YY and XL) will assess the risk of bias with the Cochrane Collaboration's tool for risk of bias 


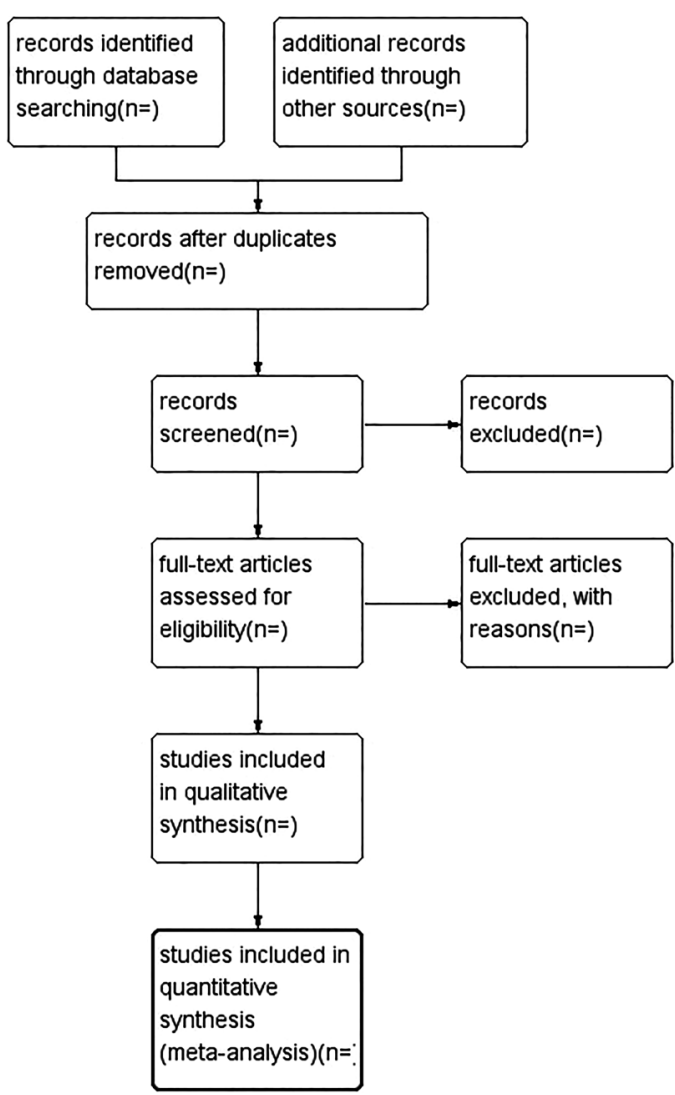

Figure 1 Study flow diagram.

assessment for all included studies. We will evaluate the following domains for risk of bias: sequence generation, allocation sequence concealment, blinding of participants and personnel and outcome assessors, incomplete outcome data, selective outcome reporting and other sources of bias. The assessments will be classified into three levels: low risk, high risk and unclear risk.

\section{Measures of treatment effect}

We will use RevMan V.5.2 for data analysis and quantitative data synthesis. For continuous data, we will use the mean difference (MD) or standard MD (SMD) to measure the treatment effect with $95 \%$ CIs if no heterogeneity is found. If significant heterogeneity is detected, the random-effects model will be used. For dichotomous data, we will use a risk ratio (RR) with 95\% CIs for analysis.

\section{Unit of analysis issues}

We will include data from parallel group-designed studies for meta-analysis. Only the first phase data will be considered in randomised cross-over trials. In these trials, participants are individually randomised to two intervention groups, and a single measurement for each outcome from each participant is collected and analysed.

\section{Dealing with missing data}

We will try to contact the first or corresponding authors of the included studies to retrieve missing or insufficient trial data. An intention-to-treat analysis will be performed, if possible (the analysis should include the data of all the participants in the groups to which they were originally randomly assigned), and a sensitivity analysis will be used to determine whether the results are inconsistent.

\section{Assessment of heterogeneity}

We will use the $\mathrm{I}^{2}$ statistic for quantifying inconsistencies among the included studies. When the $\mathrm{I}^{2}$ value is less than $50 \%$, the study will not be considered to have heterogeneity. When the $\mathrm{I}^{2}$ value exceeds $50 \%$, significant statistic heterogeneity exists among the trial and meta-analysis will not be performed. Subgroup analysis will be conducted to explore possible causes.

\section{Assessment of reporting biases}

We will use funnel plots to detect reporting biases and small-study effects. If more than 10 studies are included in the meta-analysis, we will conduct a test for funnel plot asymmetry using Egger's method. ${ }^{29}$ We will include all eligible trials, regardless of their methodological quality.

\section{Data synthesis}

Data synthesis will be conducted with RevMan V.5.2 when a meta-analysis is allowed. The results will be expressed as RR for dichotomous data and SMD for continuous data. If the $\mathrm{I}^{2}$ test is less than $50 \%$, the fixed-effects model will be used for data synthesis. If the $\mathrm{I}^{2}$ test is between $50 \%$ and $75 \%$, the random-effects model will be conducted for data synthesis. If the $\mathrm{I}^{2}$ test is higher than $75 \%$, we will investigate possible reasons from both clinical and methodological perspectives, and provide a descriptive analysis or conduct subgroup analysis.

\section{Subgroup analysis}

There is no presubgroup plan. If data are available, factors like subgroups of the chronic urticaria and acupuncture methods will be taken into account. When significant heterogeneity exists, we will also conduct subgroup analysis if necessary.

\section{Sensitivity analysis}

We will conduct sensitivity analysis to test the robustness of the primary decisions of the review process. The principal decision nodes conclude methodological quality, sample size and the effect of missing data. The meta-analysis will be repeated and studies of lower quality will be excluded. The result will be compared and discussed according to the results.

\section{Grading the quality of evidence}

The quality of evidence for all outcomes will be judged using the Grading of Recommendations Assessment, Development and Evaluation working group methodology. The following domains will be assessed: risk of 
bias, consistency, directness, precision, publication bias and additional points. The assessments will be classified into four levels: high, moderate, low or very low. ${ }^{30}$

\section{Ethics and dissemination}

A formal ethical approval is not necessary because the data that will be used are not individual and no privacy will be involved. The results will be disseminated through peer-reviewed publications or conference presentations.

\section{DISCUSSION}

Studies have signified that acupuncture is effective for relieving the symptoms (mainly in pruritus and wheals) of chronic urticaria. ${ }^{17} 31$ Nevertheless, currently no systematic review related to acupuncture for chronic urticaria has been published in English. The evaluation of this systematic review will be divided into four sections: identification, study inclusion, data extraction and data synthesis. We hope that this review will give more convincing proof to assist clinicians during the decision-making process when dealing with chronic urticaria. This review has some potential limitations. Different types of acupuncture therapies and the efficacy evaluation criteria of urticaria in included trials may cause significant heterogeneity.

PRISMA-P Checklist of the protocol: Online supplementary appendix 1

Acknowledgements The authors would like to thank Edanz Editing China for their editing work. They also thank Jing Zhou for her assistance to improve the paper.

Contributors ZL is the guarantor. QY and ZL contributed to the conception of the study. The manuscript of the protocol was drafted by $Q Y$ and revised by ZL. QY and ZQ will independently screen the potential studies and extract data from the included studies. YY and XL will assess the risk of bias and finish data synthesis. ZL will arbitrate any disagreements and ensure that no errors occur during the review. All authors read, provided feedback and approved the final manuscript.

Competing interests None declared.

Provenance and peer review Not commissioned; externally peer reviewed.

Open Access This is an Open Access article distributed in accordance with the Creative Commons Attribution Non Commercial (CC BY-NC 4.0) license, which permits others to distribute, remix, adapt, build upon this work noncommercially, and license their derivative works on different terms, provided the original work is properly cited and the use is non-commercial. See: http:// creativecommons.org/licenses/by-nc/4.0/

\section{REFERENCES}

1. Zuberbier T, Aberer W, Asero R, et al. The EAACI/GA(2) LEN/EDF/ WAO guideline for the definition, classification, diagnosis, and management of urticaria: the 2013 revision and update. Allergy 2014;69:868-87.

2. Zuberbier T, Asero R, Bindslev-Jensen $C$, et al. EAACI/GA(2)LEN/ EDF/WAO guideline: definition, classification and diagnosis of urticaria. Allergy 2009;64:1417-26.

3. Zuberbier T, Bindslev-Jensen C, Canonica W, et al. EAACl/ GA2LEN/EDF guideline: definition, classification and diagnosis of urticaria. Allergy 2006;61:316-20.
4. Zuberbier T, Greaves MW, Juhlin L, et al. Definition, classification, and routine diagnosis of urticaria: a consensus report. J Investig Dermatol Symp Proc 2001;6:123-7.

5. Maurer M, Weller K, Bindslev-Jensen C, et al. Unmet clinical needs in chronic spontaneous urticaria. A GA(2)LEN task force report. Allergy 2011;66:317-30.

6. Maurer M, Ortonne JP, Zuberbier T. Chronic urticaria: an internet survey of health behaviours, symptom patterns and treatment needs in European adult patients. Br J Dermatol 2009;160:633-41.

7. Delong LK, Culler SD, Saini SS, et al. Annual direct and indirect health care costs of chronic idiopathic urticaria: a cost analysis of 50 nonimmunosuppressed patients. Arch Dermatol 2008;144:35-9.

8. Marasoglu Celen O, Kutlubay Z, Aydemir EH. Usefulness of the autologous serum test for the diagnosis of chronic idiopathic urticaria. Ann Dermatol 2014;26:592-7.

9. Maurer M, Magerl M, Metz M, et al. Revisions to the international guidelines on the diagnosis and therapy of chronic urticaria. J Dtsch Dermatol Ges 2013.

10. Zuberbier T, Asero R, Bindslev-Jensen C, et al. EAACl/GA(2)LEN/ EDF/WAO guideline: management of urticaria. Allergy 2009;64:1427-43.

11. Sharma M, Bennett C, Cohen SN, et al. H1-antihistamines for chronic spontaneous urticaria. Cochrane Database Syst Rev 2014;11:CD006137.

12. Ridolo $\mathrm{E}$, Montagni $\mathrm{M}$, Bonzano $\mathrm{L}$, et al. Bilastine: new insight into antihistamine treatment. Clin Mol Allergy 2015;13:1.

13. Young MM, Stevens A, Porath-Waller A, et al. Effectiveness of brief interventions as part of the screening, brief intervention and referral to treatment (SBIRT) model for reducing the non-medical use of psychoactive substances: a systematic review protocol. Syst Rev 2012;1:22

14. Simons FE, Simons KJ. Histamine and H1-antihistamines: celebrating a century of progress. J Allergy Clin Immunol 2011:128:1139-50.e4.

15. Yuan Chen HZ, Hou T, Feng M. Advances in clinical researches on acupuncture treatment of chronic urticaria. 2005.

16. Park JS, Lee MJ, Chung $\mathrm{KH}$, et al. Live bee acupuncture (Bong-Chim) dermatitis: dermatitis due to live bee acupuncture therapy in Korea. Int J Dermatol 2013;52:1519-24.

17. Chen CJ, Yu HS. Acupuncture treatment of urticaria. Arch Dermatol 1998;134:1397-9.

18. Chen CJ, Yu HS. Acupuncture, electrostimulation, and reflex therapy in dermatology. Dermatol Ther 2003;16:87-92.

19. Tang C. Study on therapeutic effect and mechanism of acupuncture in the treatment of chronic urticaria [master's thesis]. Dalian Medical University, 2006.

20. Xie Q, Li S. The anti-histamine effect of acupuncture. Acupunct Res 1985:10:15-20.

21. Li B, Shi L, Du YH, et al. Acupuncture and moxibustion for chronic urticaria: a systematic review. J Tradit Chin Med 2009;50:432-6.

22. Dermatology CSo. Chinese guidelines for the diagnosis and treatment of urticaria version 2007. Chin J Dermatol 2007;40:591-3.

23. Dermatology IgoCSo. Chinese guidelines for the diagnosis and treatment of urticaria version 2014. Chin J Dermatol 2014;47:514-16.

24. Jiang Y, Yin L, Wang Y, et al. Assessments of different kinds of sham acupuncture applied in randomized controlled trials. $J$ Acupunct Tuina Sci 2011;9:199-203.

25. Fei Y, Guo Z, Chai Q, et al. Methodology key points and quality control in randomized controlled trials of needling. J Beijing Univ Tradit Chin Med 2013(02):27-30

26. Mlynek A, Zalewska-Janowska A, Martus $P$, et al. How to assess disease activity in patients with chronic urticaria? Allergy 2008;63:777-80.

27. O'Donnell BF. Urticaria: impact on quality of life and economic cost. Immunol Allergy Clin North Am 2014;34:89-104.

28. Higgins JPT, Green S, eds. Cochrane handbook for systematic reviews of interventions version 5.1.0 [updated March 2011]. The Cochrane Collaboration, 2011. http://www.cochrane-handbook.org

29. Deeks JJ, Higgins JPT, Altman DG. Analysing data and undertaking meta-analysis. In: Higgins JPT, Green S, eds. Cochrane handbook for systematic reviews of interventions version 5.1.0 [updated March 2011]. The Cochrane Collaboration, 2011. http://www. cochrane-handbook.org (accessed Jun 2014).

30. The GRADE Working Group. List of GRADE working group publications and grants. http://www.gradeworkinggroup.org/ publications/index.htm

31. Chen $\mathrm{Y}$, Zheng $\mathrm{H}$, Hou T, et al. Advances in clinical researches on acupuncture treatment of chronic urticaria [in Chinese]. Lishizhen Med Mater Med Res 2011:2047-8. 